\title{
NEURORADIOLOGICAL, NEUROPHYSIOLOGICAL AND MOLECULAR FINDINGS IN INFANTILE KRABBE DISEASE: TWO CASE REPORTS
}

\author{
Vargiami $\mathrm{E}^{1}$, Papathanasiou $\mathrm{E}^{1}$, Batzios $\mathrm{S}^{1}$, Kyriazi $\mathrm{M}^{1}$, Dimitriou $\mathrm{E}^{2}$,
} Anastasiou $\mathrm{A}^{3}$, Michelakakis $\mathrm{H}^{2}$, Giese A-K ${ }^{4}$, Zafeiriou DI ${ }^{1, *}$

\begin{abstract}
*Corresponding Author: Dimitrios I. Zafeiriou, M.D., Ph.D., Professor in Child Neurology and Developmental Pediatrics, $1^{\text {st }}$ Department of Pediatrics, Aristotle University of Thessaloniki, Egnatia St. 106, 54622 Thessaloniki, Greece. Tel./Fax: +30-2310-241-845. Mobile: +30-6944-330-587. E-mail: jeff@med.auth.gr
\end{abstract}

\begin{abstract}
Krabbe disease is an autosomal recessive neurodegenerative disorder due to a defect of the lysosomal enzyme $\beta$-galactocerebrosidase ( $\beta$-GALC). Depending on the age of onset, the disease is classified into infantile and later-onset forms. We report neuroradiological, neurophysiological and molecular findings in two Greek patients with the infantile form of Krabbe disease. The index patients presented at the age of 3.5 and 6 months, respectively, due to developmental delay. Magnetic resonance imaging (MRI) of the first patient's brain demonstrated signs of leukodystrophy, while nerve conduction velocities (NCVs) were significantly decreased. The second patient's MRI at the age of 4 months was initially normal, but at 18 months demonstrated leukodystrophic alterations as well, whereas NCVs were also significantly delayed. In both patients, a severe decrease in $\beta$-GALC, activity supported the diagnosis of Krabbe disease, while the final diagnosis was confirmed by molecular genetic testing. Two homozygous mutations of the GALC gene, the c.411_413delTAA [p.K139del] mutation in
\end{abstract}

\footnotetext{
${ }^{1} 1^{\text {st }}$ Pediatric Clinic, Aristotle University of Thessaloniki, Thessaloniki, Greece

${ }^{2}$ Department of Enzymology and Cellular Function, Institute of Child Health, Athens, Greece

${ }^{3}$ Department of Radiology, Hippokratio General Hospital, Thessaloniki, Greece

${ }^{4}$ Neurogenetics and Metabolic Disorders Unit, University of Rostock, Rostock, Germany
}

the first patient, and the c.749T $>$ C [p.I250T] mutation in the second patient, were identified. At their last follow-up visit at the age of 4 and 6 years, respective$1 y$, both patients were bedridden and quadri-plegic, suffering from frequent respiratory tract infections and fed through a gastrostomy. Both mutations found in homozygosity in these two unrelated patients of Greek ancestry, could pinpoint a common origin. Genotyping of patients with Krabbe disease is important, in order to contribute to the creation of a European mutation database and to further study possible genotype-phenotype correlations of the disease.

Keywords: $\beta$-galactocerebrosidase ( $\beta$-GALC); GALC gene; Krabbe disease; Leukodystrophy; Mutation; Nerve conduction velocity (NCV)

\section{INTRODUCTION}

Krabbe disease or globoid-cell leukodystrophy, is an autosomal recessive lysosomal disorder that affects the white matter of both central and peripheral nervous systems. It is the result of a deficiency of the lysosomal enzyme $\beta$-galactocerebrosidase (galactosylceramidase, $\beta$-GALC or, in very few cases, it is due to lack of activator saposin A (sphingolipid activator protein) [1-3]. The gene encoding $\beta$-GALC has been mapped to $14 \mathrm{q} 31$. This gene, located on chromosome $14 \mathrm{q} 31$, encodes the $\beta$-GALC protein, which has a hydrolase function critical for glycosphingolipid catabolism. To date, more than 130 mutations of the GALC gene have been listed in the Human Gene Mutation Database (HGMD) [1-3]. The 
early infantile form of the Krabbe disease is the most common (85.0-90.0\% of all cases), with onset in the early infantile period (before the age of 6 months), and a clinical picture characterized by hyperirritability and psychomotor deterioration often associated with episodes of fever, while many patients develop peripheral neuropathy with decreased nerve conduction velocities (NCVs) [1-3]. We report clinical, neuroradiological, neurophysiological and molecular findings of two Greek patients with the infantile form of Krabbe disease.

Case Reports. The first patient was admitted at the age of 3.5 months, due to generalized hypertonia. $\mathrm{He}$ is the fourth child of healthy, non consanguineous parents of Caucasian origin. One of his siblings, a male infant died at the age of 7 months, presumably due to cerebral atrophy and epilepsy, without a specific diagnosis. The other two siblings are healthy females, 13 and 8 years old, respectively.

The patient was delivered normally after an uneventful gestation at 40 weeks. Generalized hypertonia was evident already from the first month of life, according to the family pediatrician.

Physical examination on admission revealed short stature (below the 3rd percentile), severe hypertonia of all extremities, absence of head control, opisthotonus, fisted hands and pyramidal tract signs with increased deep tendon reflexes, positive Rossolimo reflexes and clonus bilaterally. Additionally, a mild convergent squint was noted, with normal reaction to visual and auditory stimuli.

Magnetic resonance imaging (MRI) of the patient's brain demonstrated abnormal signal of the deep white matter of the cerebellum, the middle cerebellar peduncles and probably the pyramidal tracts of the medulla oblongata. Additionally, there was a mild enlargement of the subarachnoid spaces and an increased signal of the posterior limbs of the internal capsule bilaterally and the white matter of the

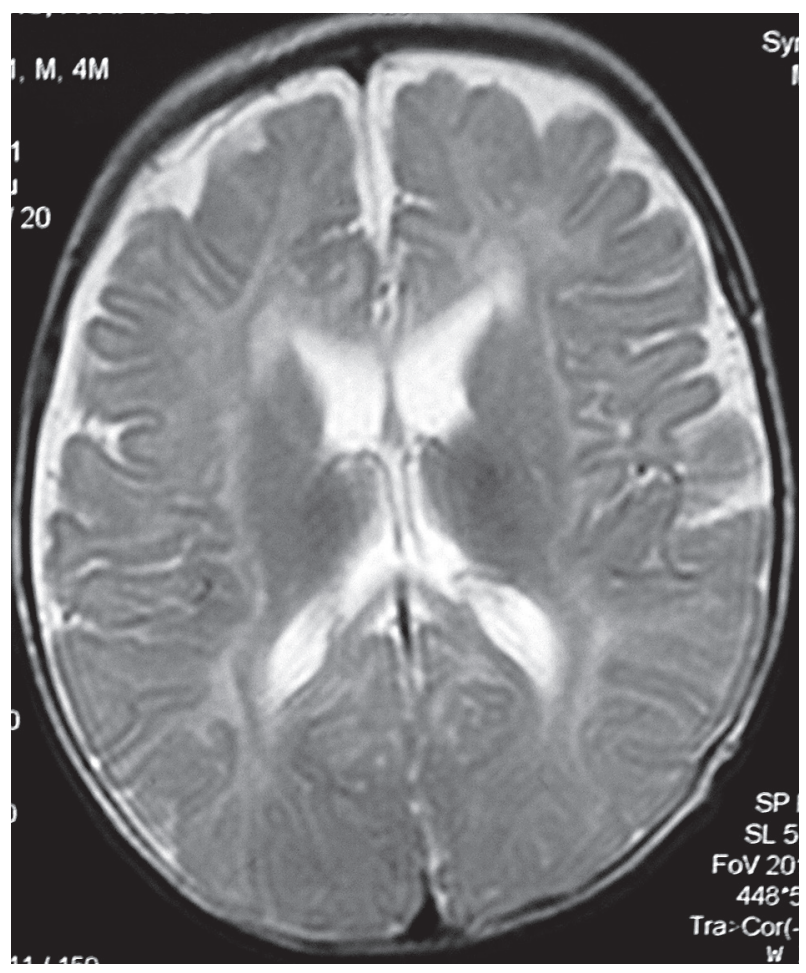

Figure 1. Brain MRI of the first patient at the age of 3.5 months (T2 weighted sequences TR/TE 4000/99). Note the mildly increased signal in the posterior limbs of the internal capsule bilaterally and in the white matter of the occipital lobe.

occipital lobes (Figure 1). Electroencephalogram, visual and brainstem auditory evoked potentials, demonstrated normal findings. Both motor and sensory NCVs were significantly decreased (Table 1) with normal compound action potentials, suggestive of demyelination.

Cerebrospinal fluid (CSF) protein was clearly elevated $(80 \mathrm{mg} / \mathrm{dL}$; normal values $<10)$. A subsequent screening for lysosomal enzymes in leukocytes (at the Department of Enzymology, Institute of Child Health, Athens, Greece) demonstrated increased chitotriosidase activity $(1566 \mathrm{nmol} / \mathrm{mL} /$ hour, normal range

Table 1. Nerve conduction velocities in both patients.

\begin{tabular}{|l|c|c|c|c|c|c|}
\hline \multicolumn{2}{|c|}{} & \multicolumn{5}{c|}{ Nerve Conduction Velocities (m/s) } \\
\hline & $\begin{array}{c}\text { Age When } \\
\text { Examined }\end{array}$ & $\begin{array}{c}\text { Median } \\
\text { Nerve }\end{array}$ & $\begin{array}{c}\text { Ulnar } \\
\text { Nerve }\end{array}$ & $\begin{array}{c}\text { Tibial } \\
\text { Nerve }\end{array}$ & $\begin{array}{c}\text { Peroneal } \\
\text { Nerve }\end{array}$ & $\begin{array}{c}\text { Sural } \\
\text { Nerve }\end{array}$ \\
\hline Patient 1 & 4 months & 28.2 & 25.0 & 21.4 & 18.7 & 33.2 \\
\hline Normal values for 1-6 months & & 33.3 & 35.0 & 35.0 & 38.8 & 40.6 \\
\hline Patient 2 & 18 months & 30.2 & 31.8 & 29.2 & 34.6 & 29.4 \\
\hline Normal values for 1-2 years & & 53.6 & 52.2 & 50.2 & 51.4 & 53.0 \\
\hline
\end{tabular}

Normal values (represent the lower normal limit [mean \pm 2 standard deviation (SD)] for NCV at the age of 4 months in our laboratory. 
$0-150)$ and a severe reduction of $\beta$-GALC activity $(0.003 \mathrm{nmol} / \mathrm{mg}$ protein/hour, normal range $0.1-0.97)$, whereas the activities of $\alpha-\mathrm{N}$-acetylglycosaminidase, $\beta$-galactosidase and $\beta$-hexosaminidase were normal.

The above biochemical findings were suggestive of Krabbe disease, a diagnosis that was confirmed by molecular genetic testing (at the Department of Genetics, Universitat de Barcelona \& Neurogenetics, Barcelona, Spain, and the Metabolic Disorders Unit, University of Rostock, Rostock, Germany). The latter revealed a homozygous c.411_413 delTAA [p.K139del] deletion on the GALC gene.

The patient's further course was downhill with further deterioration. At the last follow-up visit at the age of 4 years, he was bed-ridden and quadriplegic, suffering from frequent infections of the respiratory tract and fed through a gastrostomy catheter. Moreover, he demonstrated uncoordinated eye movements without visual contact.

The second patient was transferred to a tertiary pediatric hospital at the age of 18 months, due to generalized hypertonia and seizures. He is the only child of healthy, non consanguineous parents of Caucasian origin delivered normally after an uneventful 40 weeks' gestation. His psychomotor development was normal until the age of 6 months, when he started deteriorating in terms of motor skills, and 4 months later, a generalized hypertonia was evident. The brain MRI showed mild enlargement of both Sylvian fissures, the subarachnoid spaces of the frontal convexities and the interhemispheric fissure, as well as an increased signal of the periventricular white matter. According to the neuroradiologist, the constellation of the above findings were compatible with a delayed myelination; however, the possibility of a leukoencephalopathy could not be entirely ruled out. Due to the above findings, physical and occupational therapy were initiated.

At the age of 18 months, he manifested a generalized status epilepticus. On admission he was febrile, somnolent and had mild respiratory distress. Physical examination revealed tonsillitis. Kernig and Brudzinski signs were negative. Neurological examination revealed generalized hypertonia with fisted hands, absent pupillary light reflex, response solely to painful stimuli, nystagmus, increased deep tendon reflexes, positive Babinski sign, Rossolimo reflex and clonus bilaterally.

Examination of the fundus revealed nonresponsive pupils without papilledema, while the electroencepha-

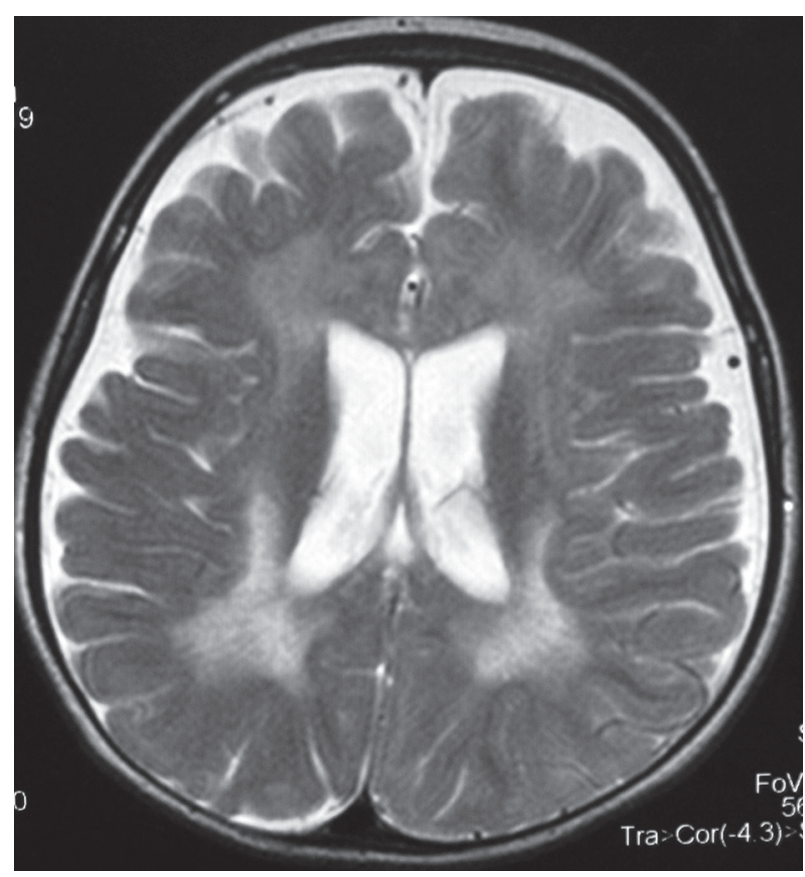

Figure 2. Brain MRI of second patient at the age of 18 months (T2 weighted sequences TR/TE 4510/119). Note the increased signal in the periventicular white matter around the occipital horns of the lateral ventricles with enlargement of both lateral ventricles and the subarachnoid spaces.

logram disclosed multifocal spikes and sharp waves. Brainstem auditory evoked and visual evoked potentials were normal. Motor and sensory NCVs were significantly delayed with normal compound action potentials, consistent with peripheral demyelination (Table 1).

A repeated brain MRI performed at the time (Figure 2), demonstrated increased signal of the white matter around the occipital horns of the lateral ventricles with enlargement of lateral ventricles and the subarachnoid spaces bilaterally. These lesions of the white matter expanded radially, while additional small lesions in the anterior fiber bundles of the pons and the brainstem.

Cerebrospinal fluid protein at that time point was clearly elevated $(110 \mathrm{mg} / \mathrm{dL}$; normal values $<10)$. A subsequent screening for lysosomal enzymes in leukocytes (at the Department of Enzymology, Institute of Child Health, Athens, Greece) demonstrated mildly increased chitotriosidase activity $(217 \mathrm{nmol} / \mathrm{nl} /$ hour, normal range $0-150$ ) and a total lack of $\beta$-GALC activity $(0.00 \mathrm{nmol} / \mathrm{mg}$ protein $/ \mathrm{hr}$, normal range 0.1 0.97 ), whereas the activities of arylsulphatase $A$, $\beta$-galactosidase and $\beta$-hexosaminidase were normal. 
The total lack of $\beta$-GALC activity was confirmed in a second assay, thus pointing to the diagnosis of Krabbe disease. The final diagnosis was established by further molecular genetic testing (at the Department of Genetics, Universitat de Barcelona \& Neurogenetics, Barcelona, Spain, and the Metabolic Disorders Unit, University of Rostock, Rostock, Germany), which revealed the presence of the a homozygous p.I250T (c.749T $>$ C) mutation on the GALC gene, with both parents being carriers.

On follow-up at 6 years of age, the patient was bedridden and quadriplegic with optic atrophy and contractures of the lower extremities, despite receiving regular physical therapy. His seizure disorder was well controlled under treatment with levatiracetam.

\section{DISCUSSION}

Krabbe disease (also known as globoid cell leukodystrophy, GCL; OMIM \#245200) is a rare inherited lysosomal storage disorder, with a pathology not completely elucidated. It is caused by a deficiency of $\beta$-GALC (E.C. 3.2.1.46), a lysosomal enzyme involved in the catabolism of galactosylceramide. The deficiency of $\beta$-GALC impairs the degradation of a major myelin lipid, galactocerebroside with the subsequent accumulation of a cytotoxic compound, galactosylsphingosine, also called psychosine [4]. The excess of galactosylceramide elicits the formation of multinucleated macrophages, the globoid cells. Progressive accumulation of psychosine could explain the prominent death of oligodendrocytes, thus contributing to progressive demyelination [5].

Krabbe disease is usually diagnosed before the first year of life, because of the early onset of developmental delay or regression, limb spasticity and extreme irritability [2]. Optic atrophy and sluggish pupillary reactions to light are common [2,6,7]. Children with Krabbe disease generally experience severe neurological deterioration and death before 2 years of age, although prolonged survival has been reported.

Both index cases could be classified as infantile onset globoid-cell leukodystrophy [2,5], with a disease onset at 3.5 and 6 months of age, respectively, and a rather typical constellation of clinical findings (developmental delay with subsequent regression, spasticity and optic atrophy) and neuroradiological findings (deep white matter leuko-dystrophic alterations). However, it is worth noticing that the second patient's brain MRI at presentation was deceptively normal, a finding already reported in the literature [8], underpinning the need for further biochemical work-up, even in the absence of associated brain MRI findings, in infants with unexplained developmental delay and/or regression.

Multimodal evoked potentials in the early infantile form of Krabbe disease are considerably abnormal at pronounced stages of the disease $[1,5,8]$. In both reported patients, brainstem auditory and visual evoked potentials were normal at the age of diagnosis. On the contrary, decreased NCVs of all examined nerves (motor and sensory) was demonstrated, without however reaching the markedly reduced values observed in late infantile Krabbe disease [9]. To the best of our knowledge, serial NCV findings in early-infantile Krabbe disease have previously been reported in another Greek case by Zafeiriou et al. [8], while a mild increase in polyphasic motor unit potentials, as demonstrated by electromyography, was reported by Hogan et al. [10].

Most patients with Krabbe disease, irrespective of type and age at onset, demonstrate a severe deficiency in $\beta$-GALC activity, as measured in leukocytes isolated from whole blood or alternatively in cultured skin fibroblasts [11]. Since the cloning of the human GALC gene on chromosome 14q31 in 1993 [2], over 130 mutations have been described [2,12]. Different kinds of genetic alterations have been reported: missense/nonsense mutations, deletions, splice mutations, insertions and duplications [2]. According to one of the largest genotypic analyses of the GALC gene performed in a European cohort of patients with Krabbe disease, the GALC mutational profile in European patients differs from other cohorts; thus, the most common large deletion, c. $1161+6532$ plus three additional mutations [c. $1586 \mathrm{C}>\mathrm{T}, \mathrm{c} .1700 \mathrm{C}>\mathrm{T}$, c.1472delA (1538 C>T, 1652A >C, 1424delA)], together account for about $60.0 \%$ of patients of European origin with the classic infantile form [11].

In the index patients, the mutation analysis of the $G A L C$ gene revealed two different mutations in a homozygous state, the c.749T $>C$ [p.I250T] mutation and the c.411_413 delTAA [K139del] mutation. A thorough literature search disclosed five patients carrying the c.749T $>$ C [p.I250T] mutation. In the first of those patients, classified as having early infantile Krabbe disease, the latter mutation was found in a heterozygous state and associated with the rare find- 
ing of optic nerve enlargement [13], as well as in two other patients associated with neonatal and infantile onset of the disease, respectively [12]. The above mutation in homozygosity was associated with late infantile Krabbe disease, in a female patient born to consanguineous parents and manifesting with motor regression and peripheral neuropathy $[8,13]$, as well as in four additional patients with early-onset disease [10]. Interestingly, some of these patients were of Greek ancestry [11,12], as our index cases.

Regarding the second mutation, the c.411_413delTAA [K139del], it has recently been described in two Greek siblings with late-onset slowly progressive disease [12]. Moreover, the same mutation has been described in a heterozygous state in an adult patient of Turkish origin [5]. These data allow the assumption that the latter mutation in a homozygous state could be associated with more severe and earlier-onset forms of the disease, while the heterozygous state could be associated with a later-onset and more protracted clinical course.

Further studies are needed in order to elucidate the genetic landscape and the clinical importance of specific mutations, as a genotype-phenotype correlation relationship in Krabbe disease still remains unclear. This would make it possible to be able to offer more accurate genetic counseling and suggest either prenatal diagnosis or pre-implantation genetics to the affected families [14].

In conclusion, we have presented brain MRI, neuro-physiological and genetic findings in two additional Greek cases of early-infantile Krabbe disease. The above report with the identified mutations add to the pathogenic mutation database of the GALC gene and may increase public awareness of Krabbe disease in Greece and elsewhere. Genotyping of patients with Krabbe disease is important, in order to contribute to the development of a European mutation database and to further study possible genotype-phenotype correlations of the disease.

\section{ACKNOWLEDGMENTS}

We would like to thank Irini Mavridou (Institute of Child Health, Athens, Greece), Lluisa Vilageliou and David Grinberg (Department of Genetics, Faculty of Biology, Universitat de Barcelona, IBUB, Ciberer, Barcelona, Spain) for their help with the molecular investigations. We are grateful to Professor Arndt
Roelfs (Neurogenetics and Metabolic Disorders Unit, University of Rostock, Rostock, Germany) for his critical review of the manuscript.

Declaration of Interest. The authors report no conflicts of interest. The authors alone are responsible for the content and writing of this article.

\section{REFERENCES}

1. Spiegel R, Bach G, Sury V, Mengistu G, Meidan $\mathrm{B}$, Shalev $\mathrm{S}$, et al. A mutation in the saposin A coding region of the prosaposin gene in an infant presenting as Krabbe disease: First report of saposin A deficiency in humans. Mol Genet Metab. 2005; 84(2): 160-166.

2. Graziano AC, Cardile V. History, genetic, and recent advances on Krabbe disease. Gene. 2015; 555(1): 2-13.

3. Szymanska K, Lugowska A, Laure-Kamionowska M, Bekiesinska-Figatowska M, GieruszczakBialek D, Musielak M, et al. Diagnostic difficulties in Krabbe disease: A report of two cases and review of literature. Folia Neuropathol. 2012; 50(4): 346-56.

4. Suzuki K. Twenty five years of the "psychosine hypothesis": A personal perspective of its history and present status. Neurochem Res. 1998; 23(3): 251-259.

5. Debs R, Froissart R, Aubourg P, Papeix C, Douillard C, Degos B, et al. Krabbe disease in adults: Phenotypic and genotypic update from a series of 11 cases and a review. J Inherit Metab Dis. 2013; 36(5): 859-868.

6. van der Knaap MS, Valk J. Globoid cell leukodystrophy (Krabbe disease). In: van der Knaap M, Valk J, Eds. Magnetic Resonance of Myelination and Myelin Disorders, 3rd ed. Berlin, Germany: Springer-Verlag. 2005: 87-95.

7. Kolodny EH, Raghavan S, Krivit W. Late-onset Krabbe disease (globoid cell leukodystrophy): Clinical and biochemical features of 15 cases. Dev Neurosci. 1991; 13(4-5): 232-239.

8. Zafeiriou DI, Anastasiou AL, Michelakaki EM, Augoustidou-Savvopoulou PA, Katzos GS, Kontopoulos EE. Early infantile Krabbe disease: Deceptively normal magnetic resonance imaging 
and serial neurophysiological studies. Brain Dev. 1997; 19(7): 488-491.

9. Zafeiriou DI, Michelakaki EM, Anastasiou AL, Gombakis NP, Kontopoulos EE. Serial MRI and neuro-physiological studies in late-infantile Krabbe disease. Pediatr Neurol. 1996; 15(3): 240-244.

10. Hogan GR, Gutmann L, Chou SM. The peripheral neuropathy of Krabbe's (globoid) leukodystrophy. Neurology. 1969; 19(11): 1093-1097.

11. Tappino B, Biancheri R, Mort M, Regis S, Corsolini F, Rossi A, et al. Identification and characterization of 15 novel GALC gene mutations causing Krabbe disease. Hum Mutat. 2010; 31(12): E1894-E1914.
12. Dimitriou E, Cozar M, Mavridou I, Grinberg D, Vilageliu L, Michelakakis H. The spectrum of Krabbe disease in Greece: Biochemical and molecular findings. JIMD Rep. 2015 Jun 25. [Epub ahead of print] doi: 10. 1007/j.jimdrep.8904_2015_457.

13. Hussain SA, Zimmerman HH, Abdul-Rahman OA, Hussaini SM, Parker SS, Khan M, et al. Optic nerve enlargement in Krabbe disease: A pathophysiologic and clinical perspective. J Child Neurol. 2011; 26(5): 642-644.

14. Kardas F, Uzak AS, Hossain MA, Sakai N, Canpolat M, YikılmazA. A novel homozygous GALC mutation: Very early onset and rapidly progressive Krabbe disease. Gene. 2013; 517(1): 125-127. 